\title{
SimpleBounce: A simple package for the false vacuum decay
}

\section{Ryosuke Sato}

Deutsches Elektronen-Synchrotron (DESY), Notkestraße 85, D-22607 Hamburg, Germany

\section{A R T I C L E I N F O}

\section{Article history:}

Received 6 January 2020

Received in revised form 21 July 2020

Accepted 12 August 2020

Available online 26 August 2020

\section{Keywords:}

Phase transitions

Bounce solution

Euclidean action

\begin{abstract}
A B S T R A C T
We present SimpleBounce, a C++ package for finding the bounce solution for the false vacuum decay. This package is based on a flow equation which is proposed by the author R. Sato (2020) and solves Coleman-Glaser-Martin's reduced problem (S. R. Coleman et al. 1978): the minimization problem of the kinetic energy while fixing the potential energy. The bounce configuration is obtained by a scale transformation of the solution of this problem. For models with 1-8 scalar field(s), the bounce action can be calculated with $\mathcal{O}(0.1) \%$ accuracy in $\mathcal{O}(0.1) \mathrm{s}$. This package is available at http://github.com/rsato64/SimpleBounce.

Program summary

Program title: SimpleBounce.

CPC Library link to program files: http://dx.doi.org/10.17632/g74z22ryht.1

Developer's repository link: http://github.com/rsato64/SimpleBounce

Licensing provisions: GPLv3.

Programming language: $\mathrm{C}++$

Nature of problem: The decay rate of the false vacuum can be evaluated by using Euclidean path integral formalism, and the bounce solution is a saddle point of this path integral. The bounce solution and its Euclidean action are numerically evaluated.

Solution method: The bounce solution is obtained as a fixed point of gradient flow equation.

Additional comments including restrictions and unusual features: The computation is fast and stable against a choice of initial configuration.

References:

[1] R. Sato, Simple Gradient Flow Equation for the Bounce Solution,10.1103/PhysRevD.101.016012 Phys. Rev. D 101 no. 1, (2020) 016012, arXiv:1907.02417[hep-ph]
\end{abstract}

(C) 2020 Elsevier B.V. All rights reserved.

\section{Introduction}

The decay of the false vacua is an important phenomenon in particle physics and cosmology. The lifetime of the false vacua can be calculated by the Euclidean path integral [1]. (For an earlier work, see Ref. [2,3].) In this formalism, the bounce solution which is a saddle point of the action gives the dominant contribution to the decay width. There exist several numerical packages to calculate the bounce solution; CosmoTransitions [4,5], ${ }^{1}$ AnyBubble [6], ${ }^{2}$ and BubbleProfiler $[7,8] .{ }^{3}$ For other numerical algorithms, see Refs. [9-21].

In this paper, we introduce a new numerical package to calculate the bounce solution. Our package utilizes a gradient flow equation. This framework is recently proposed by Chigusa, Moroi, and Shoji [22]. Our package solves a flow equation which is

\footnotetext{
E-mail address: ryosukesato64@gmail.com.

1 https://clwainwright.github.io/CosmoTransitions/.

2 http://cosmos.phy.tufts.edu/AnyBubble/.

3 https://github.com/bubbleprofiler/bubbleprofiler.
}

proposed by the author [23]. As we will see in the next section, our flow equation minimizes the kinetic energy of configuration while fixing the potential energy. Thanks to Coleman-GlaserMartin's discussion [24], the bounce solution is obtained by a scale transformation of the fixed point of the flow equation.

\section{Formulation}

Here we briefly summarize the gradient flow equation which is proposed in Ref. [23].

We take the Euclidean action with $n_{\phi}$ scalar fields which have the canonical kinetic term and a generic potential term:

$$
\begin{aligned}
& \mathcal{S}_{E}[\phi]=\mathcal{T}[\phi]+\mathcal{V}[\phi], \quad \mathcal{T}[\phi]=\sum_{i=1}^{n_{\phi}} \int d^{d} x \frac{1}{2}\left(\nabla \phi_{i}\right)^{2}, \\
& \mathcal{V}[\phi]=\int d^{d} x\left[V(\phi)-V\left(\phi_{F V}\right)\right] .
\end{aligned}
$$

Here $d$ is the dimension of the Euclidean space, and we assume $d$ is larger than 2. The scalar potential $V$ satisfies $\partial V /\left.\partial \phi_{i}\right|_{\phi=\phi_{F V}}=0$, 
all of the eigenvalues of the Hessian of $V$ at $\phi_{i}=\phi_{F V, i}$ are nonnegative, and $V(\phi)-V\left(\phi_{F V}\right)$ is somewhere negative. The bounce solution is a configuration which satisfies the following equation of motion and the boundary condition at infinity:

$-\nabla^{2} \phi_{i}+\frac{\partial V}{\partial \phi_{i}}=0, \quad \lim _{|x| \rightarrow \infty} \phi_{i}(x)=\phi_{F V, i}$.

The bounce solution has $O(d)$ symmetry [24-27] in the space. Thus, the equation of motion can be simplified as

$-\frac{d^{2} \phi_{i}}{d r^{2}}-\frac{d-1}{r} \frac{d \phi_{i}}{d r}+\frac{\partial V}{\partial \phi_{i}}=0$.

Here we solve the minimization problem of the kinetic energy $\mathcal{T}[\phi]$ while fixing the negative potential energy $\mathcal{V}[\phi]<0$ rather than solving the above equation of motion directly. This is the reduced problem of the bounce solution which is proposed in Ref. [24]. The solution of this problem is a scale-transformed of the bounce solution. In Ref. [23], a gradient flow equation is proposed to solve this problem. We introduce functions $\varphi_{i}(r, \tau)$ and the flow of $\varphi$ is described as

$$
\begin{aligned}
\frac{\partial}{\partial \tau} \varphi_{i}(r, \tau) & =\nabla^{2} \varphi_{i}-\lambda[\varphi] \frac{\partial V(\varphi)}{\partial \varphi_{i}}, \\
\lambda[\varphi]= & \frac{\sum_{i=1}^{n_{\phi}} \int_{0}^{\infty} d r r^{d-1} \frac{\partial V(\varphi)}{\partial \varphi_{i}} \nabla^{2} \varphi_{i}}{\sum_{i=1}^{n_{\phi}} \int_{0}^{\infty} d r r^{d-1}\left(\frac{\partial V(\varphi)}{\partial \varphi_{i}}\right)^{2}} .
\end{aligned}
$$

Note that $\lambda[\varphi]$ depends on $\tau$ but not on $r$. Here $\tau$ is "time" for the flow of $\varphi$ and $\nabla^{2} \varphi_{i}=\partial_{r}^{2} \varphi_{i}+(d-1)\left(\partial_{r} \varphi\right) / r$. We take the initial $\varphi(r, 0)$ such that

$\left.\mathcal{V}[\varphi]\right|_{\tau=0}<0$.

We can easily check

$$
\frac{d}{d \tau} \mathcal{V}[\varphi]=0, \quad \frac{d}{d \tau} \mathcal{T}[\varphi] \leq 0
$$

For details, see Ref. [23]. In the limit of large $\tau, \varphi$ reaches a fixed point and satisfies $\nabla^{2} \varphi-\lambda[\varphi](\partial V / \partial \varphi)=0$. This fixed point is not a saddle point because we fixed $\mathcal{V}$, and this property guarantees the stability of numerical calculation. The bounce solution $\phi_{B}$ can be obtained by the following scale transformation of the fixed point $\varphi$.

$\phi_{B}(r)=\lim _{\tau \rightarrow \infty} \varphi\left(\lambda^{1 / 2} r, \tau\right)$.

Note that $\mathcal{V}\left[\phi_{B}\right]<0$ is guaranteed thanks to Eqs. (6) and (7) and $\phi_{B}$ is a configuration which is relevant with the false vacuum decay.

\section{Algorithm}

We solve the flow equation Eq. (4) numerically. We discretize $r$-space and take $n$ points at $r_{i}=(i-1) \delta r$ for $i=1, \ldots, n$. The radius of the sphere is $R \equiv(n-1) \delta r$, however, the value of $R$ itself is not important because we utilize the scale transformation property of the bounce solution. We take the following discretized Laplacian which is similar to Ref. [28]:

$$
\begin{aligned}
& \left.\nabla^{2} \varphi_{i}\right|_{r=r_{j}} \\
& \quad=\left(\frac{d^{2} \varphi_{i}}{d r^{2}}+\frac{d-1}{r} \frac{d \varphi_{i}}{d r}\right)_{r=r_{j}} \\
& \quad= \begin{cases}\frac{2 d\left(\varphi_{i, 2}-\varphi_{i, 1}\right)}{\delta r^{2}} & (j=1) \\
\frac{\varphi_{i, j+1}-2 \varphi_{i, j}+\varphi_{i, j-1}}{\delta r^{2}}+\frac{d-1}{r_{j}} \frac{\varphi_{i, j+1}-\varphi_{i, j-1}}{2 \delta r} & (j>1)\end{cases}
\end{aligned}
$$

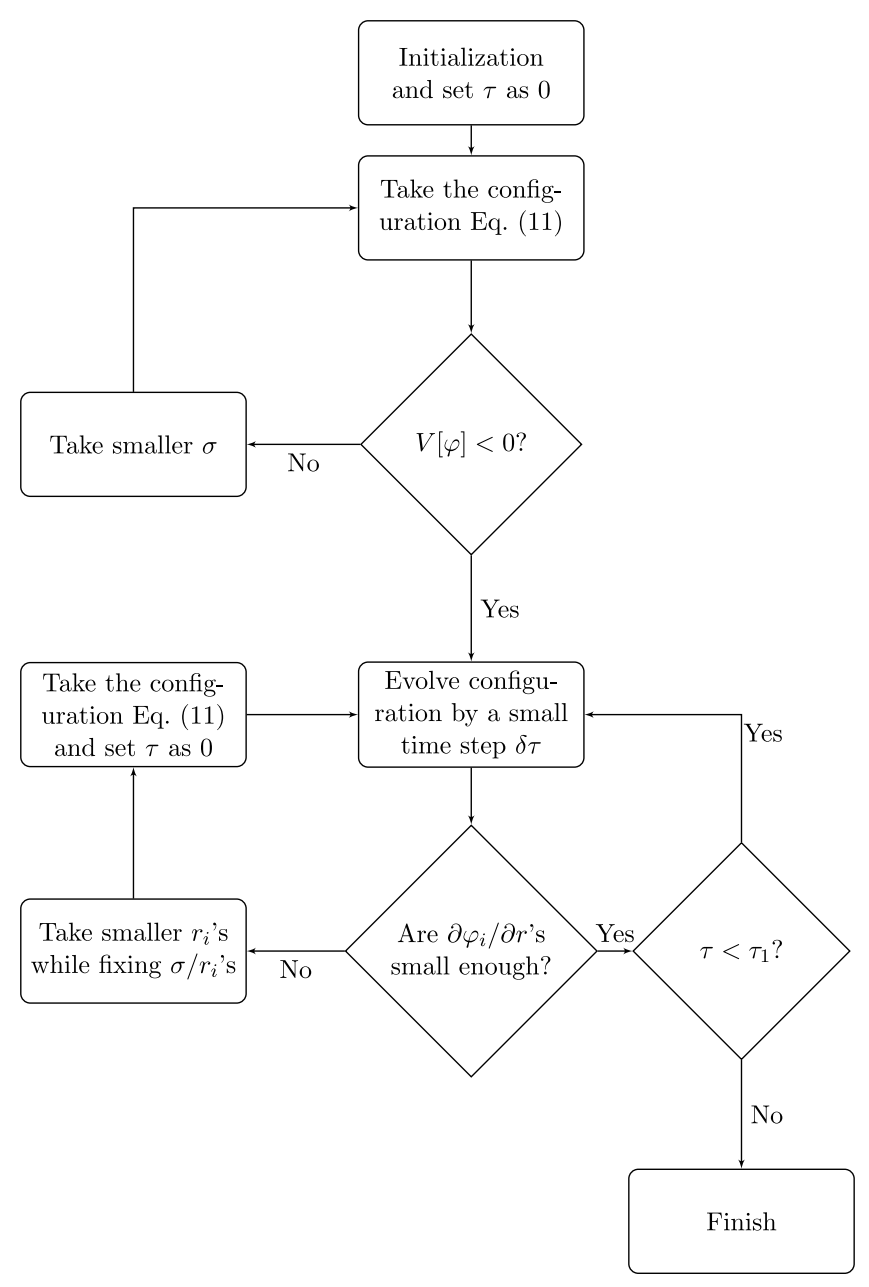

Fig. 1. Flow chart of the algorithm of SimpleBounce. The default values of $\tau_{0}$ and $\tau_{1}$ are 0.05 and 0.4 .

The integrals in Eq. (5) are approximated by the trapezoidal rule. We take a boundary condition such that $\varphi_{i, n}=\phi_{F V, i}$.

Each step of the evolution of $\varphi$ is evaluated as

$\varphi_{i}(r, \tau+\delta \tau)=\delta \tau \times\left[\nabla^{2} \varphi_{i}-\lambda[\varphi] \frac{\partial V(\varphi)}{\partial \varphi_{i}}\right]$.

We repeat this evolution until $\varphi$ converges. For the stability of the numerical calculation, $\delta \tau$ should be $\mathcal{O}\left(\delta r^{2}\right)$ otherwise the fluctuation with large wave number exponentially grows.

We take the following configuration as the initial condition.

$\varphi_{i}(r, 0)=\phi_{T V, i}+\frac{1}{2}\left(\phi_{F V, i}-\phi_{T V, i}\right)\left(1+\tanh \left(\frac{r_{i}-r_{0}}{\sigma}\right)\right)$.

Here $\phi_{T V}$ is chosen such that $V\left(\phi_{T V}\right)<V\left(\phi_{F V}\right)$. (Note that $\phi_{T V}$ is not necessarily to be the true vacuum. Also the algorithm presented here works even there exists no true vacuum. For example, Ref. [23] shows this algorithm works with $V(\phi)=$ $\phi^{2} / 2-\phi^{3} / 3$.) The initial $r_{0}$ is set to some value which is smaller than $R(0.5 R$ as default), and $\sigma$ is set to be sufficiently small to obtain negative $\mathcal{V}$. In order to satisfy the boundary condition at infinity, the first derivative of the field should be small enough. If $\partial \phi_{i} /\left.\partial r\right|_{r=R}=\left(\phi_{i, n}-\phi_{i, n-1}\right) / \delta r$ is not small after solving the flow equation, the size of the bounce is not small enough compared to the size of the space $R$. In this case, we solve the flow equation again with smaller $r_{0}$. See also Fig. 1 . 


\section{User instructions}

This package is available at https://github.com/rsato64/Simp leBounce. The main files are simplebounce.cc and simplebounce.h. The sample executable files are compiled by

make

The default compiler is $\mathrm{g}^{++}$. If you need to change this, please edit Makefile.inc. In this package, the following examples are available.

- sample1.x

This calculates the Euclidean action for the bounce in a single scalar field model. See also Section 5.1.

- benchmark/compare_with_cosmotransitions/ run_simplebounce.sh in this folder gives Table 1. See also Section 5.2 .

- benchmark/change_n_tau/

run.sh in this folder gives Fig. 2. See also Section 5.3.

\section{Sample codes and discussions}

Here we describe several sample codes and discuss their results.

\subsection{Sample code for single scalar field model}

Here we show an example of code. The following information is required to calculate the bounce solution:

- The number of scalar field $n_{\phi}$

- The scalar potential $V(\phi)$

- The first derivative(s) of the scalar potential $\partial V / \partial \phi_{i}$

- The position of the false vacuum $\phi_{F V}$

- A point $\phi_{T V}$ which gives $V\left(\phi_{T V}\right)<V\left(\phi_{F V}\right)$.

Here we take the potential as

$V(\phi)=\frac{1}{2} \phi^{2}-\frac{1}{3} \phi^{3}$.

The false vacuum is at $\phi=0$. The scalar field $\phi$ will tunnel into positive $\phi$ region. The source file sample1.cc is given as

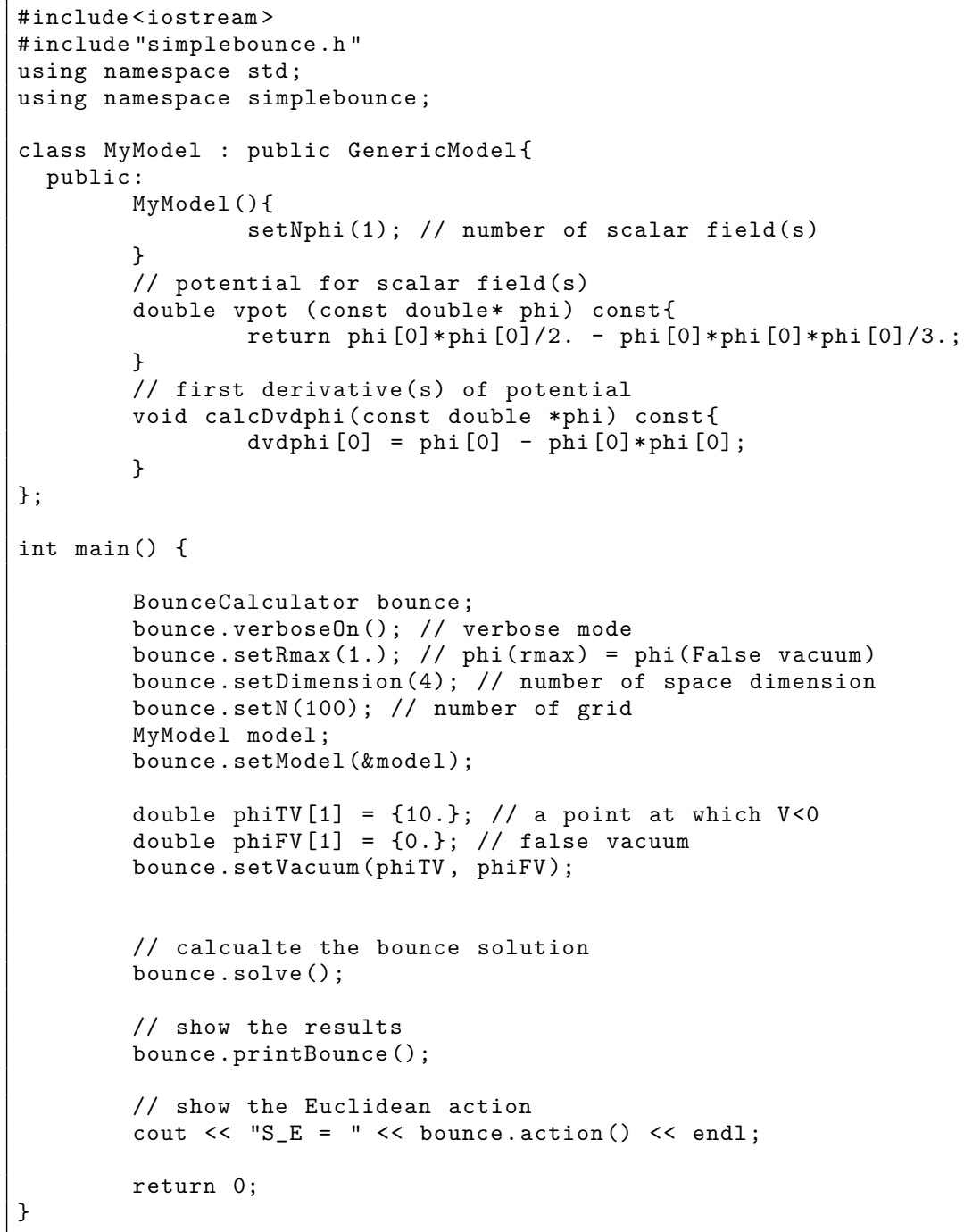


Table 1

The comparison with CosmoTransitions. For CosmoTransitions, we take fRatioConv as 0.02 . The bounce action $\mathcal{S}_{E}$ is calculated for $d=3$. The runtimes are measured by Thinkpad X250 with Ubuntu 16.04, whose CPU is Intel ${ }^{\circledR}$ Core $^{\mathrm{TM}} \mathrm{i7-5600U}(2.60 \mathrm{GHz})$ and compiler is $\mathrm{GCC}$ version 5.4.0. We take -03 as the optimization option.

\begin{tabular}{llllll}
\hline Model & \multicolumn{2}{l}{$\mathcal{S}_{E}$} & & \multicolumn{2}{l}{ Time [s] } \\
\cline { 2 - 3 } \cline { 5 - 6 } & SB & CT & & SB & CT \\
\hline \#1 in Tab. 1 of [8] & 52.4 & 52.6 & & 0.04 & 0.05 \\
\#2 in Tab. 1 of [8] & 20.8 & 21.1 & & 0.04 & 0.35 \\
\#3 in Tab. 1 of [8] & 22.0 & 22.0 & & 0.07 & 0.17 \\
\#4 in Tab. 1 of [8] & 55.8 & 56.1 & & 0.07 & 0.31 \\
\#5 in Tab. 1 of [8] & 16.2 & 16.4 & & 0.09 & 0.26 \\
\#6 in Tab. 1 of [8] & 24.4 & 24.5 & & 0.11 & 0.25 \\
\#7 in Tab. 1 of [8] & 36.6 & 36.7 & & 0.14 & 0.22 \\
\#8 in Tab. 1 of [8] & 45.9 & 46.1 & & 0.16 & 0.23 \\
Eq. 40 of [22] & $1.08 \times 10^{3}$ & $1.09 \times 10^{3}$ & & 0.03 & 0.09 \\
Eq. 41 of [22] & 6.62 & 6.65 & & 0.03 & 0.06 \\
Eq. 42 of [22] & $1.75 \times 10^{3}$ & $1.77 \times 10^{3}$ & & 0.34 & 0.54 \\
Eq. 43 of [22] & 4.45 & 4.50 & & 0.05 & 0.19 \\
\hline
\end{tabular}

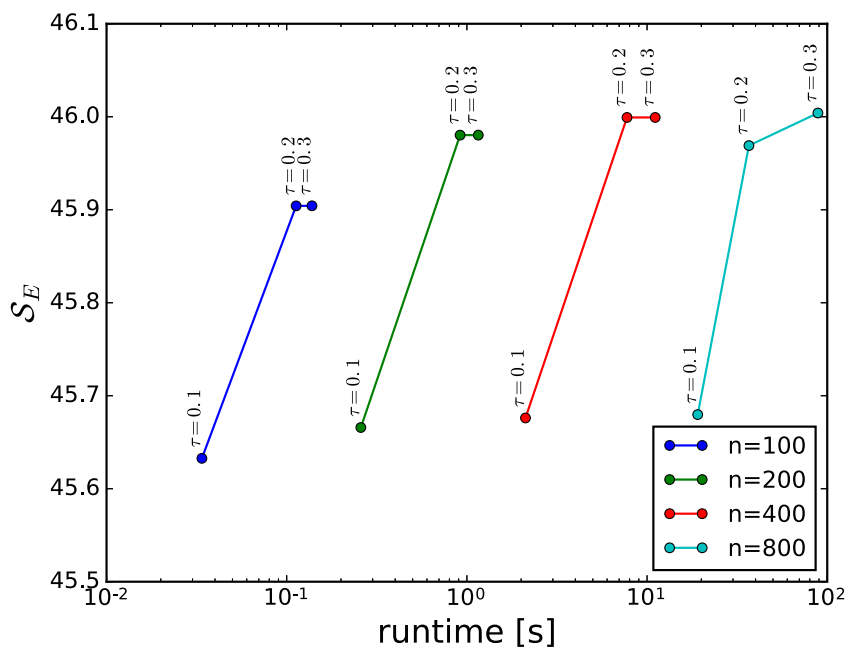

Fig. 2. The runtime of the value of $\mathcal{S}_{E}$ for different number of the lattice $n$ and the flow time $\tau_{1}$. We take the model \#8 in Tab. 1 of [8]. The environment of PC is same as Table 1.

Then, the executable file can be generated by, for example, $\mathrm{g}++$ sample1.cc simplebounce.cc -03 .

\subsection{The Euclidean action for benchmark models}

Table 1 shows the Euclidean action and the runtime for several benchmark models, which are taken from Refs. [8,22]. We also show the results and runtimes by CosmoTransitions in the table. We can see the results of our package agree with CosmoTransitions, and our package is faster for the benchmark models. We can expect the running time is proportional to the number of the scalar field because the code calculates Eqs. (10) and (5) at each time step. Table 1 is roughly consistent with this expectation.

\subsection{Changing $n$ and $\tau_{1}$}

In Fig. 2, we show the value of $\mathcal{S}_{E}$ for different number of the lattice $n$ and the flow time $\tau_{1}$. We can see that the value of $\mathcal{S}_{E}$ converges for large $n$ and large $\tau_{1}$, and we can get the result of $\mathcal{O}(0.1) \%$ accuracy in $\mathcal{O}(0.1) \mathrm{s}$ run with $n=100$. Fig. 2 also shows that the runtime is proportional to $n^{3}$. This is because the step $\delta \tau$ is bounded as $\sim \delta r^{2}$. Thus, in general, thin wall bounce takes much more time than thick wall bounce to obtain accurate result.

\section{Declaration of competing interest}

The authors declare that they have no known competing financial interests or personal relationships that could have appeared to influence the work reported in this paper.

\section{Acknowledgments}

The author thanks Tomohiro Abe, Yohei Ema, Parsa Ghorbani, Ryusuke Jinno, Thomas Konstandin, and Kyohei Mukaida for useful discussions.

\section{References}

[1] S.R. Coleman, Phys. Rev. D15 (1977) 2929-2936, Erratum:; Phys. Rev. D16 (1977) 1248.

[2] T.D. Lee, G.C. Wick, Phys. Rev. D9 (1974) 2291-2316.

[3] P.H. Frampton, Phys. Rev. Lett. 37 (1976) 1378, Erratum:; Phys. Rev. Lett. 37 (1976) 1716.

[4] S. Profumo, L. Ubaldi, C. Wainwright, Phys. Rev. D 82 (2010) 123514 arXiv: 1009.5377 [hep-ph].

[5] C.L. Wainwright, Comput. Phys. Comm. 183 (2012) 2006-2013, arXiv: 1109.4189 [hep-ph].

[6] A. Masoumi, K.D. Olum, B. Shlaer, J. Cosmol. Astropart. Phys. 1701 (01) (2017) 051, arXiv:1610.06594 [gr-qc].

[7] S. Akula, C. Balázs, G.A. White, Eur. Phys. J. C76 (12) (2016) 681, arXiv: 1608.00008 [hep-ph].

[8] P. Athron, C. Balázs, M. Bardsley, A. Fowlie, D. Harries, G. White, Comput Phys. Comm. 244 (2019) 448-468, arXiv:1901.03714 [hep-ph].

[9] M. Claudson, L.J. Hall, I. Hinchliffe, Nuclear Phys. B228 (1983) 501-528.

[10] A. Kusenko, Phys. Lett. B358 (1995) 51-55, arXiv:hep-ph/9504418 [hep-ph].

[11] A. Kusenko, P. Langacker, G. Segre, Phys. Rev. D54 (1996) 5824-5834, arXiv:hep-ph/9602414 [hep-ph].

[12] J.M. Moreno, M. Quiros, M. Seco, Nuclear Phys. B526 (1998) 489-500, arXiv:hep-ph/9801272 [hep-ph].

[13] J.M. Cline, J.R. Espinosa, G.D. Moore, A. Riotto, Phys. Rev. D59 (1999) 065014, arXiv:hep-ph/9810261 [hep-ph].

[14] P. John, Phys. Lett. B452 (1999) 221-226, arXiv:hep-ph/9810499 [hep-ph]

[15] J.M. Cline, G.D. Moore, G. Servant, Phys. Rev. D60 (1999) 105035, arXiv: hep-ph/9902220 [hep-ph].

[16] T. Konstandin, S.J. Huber, J. Cosmol. Astropart. Phys. 0606 (2006) 021 arXiv:hep-ph/0603081 [hep-ph].

[17] J.-h. Park, J. Cosmol. Astropart. Phys. 1102 (2011) 023, arXiv:1011.4936 [hep-ph].

[18] V. Guada, A. Maiezza, M. Nemevšek, Phys. Rev. D99 (5) (2019) 056020, arXiv:1803.02227 [hep-th]

[19] R. Jinno, Machine learning for bounce calculation, arXiv:1805.12153 [hep-th].

[20] J.R. Espinosa, T. Konstandin, J. Cosmol. Astropart. Phys. 1901 (01) (2019) 051, arXiv:1811.09185 [hep-th].

[21] M.L. Piscopo, M. Spannowsky, P. Waite, Phys. Rev. D100 (1) (2019) 016002 , arXiv:1902.05563 [hep-ph].

[22] S. Chigusa, T. Moroi, Y. Shoji, Phys. Lett. B 800 (2020) 135115, arXiv: 1906.10829 [hep-ph].

[23] R. Sato, Phys. Rev. D 101 (1) (2020) 016012, arXiv:1907.02417 [hep-ph].

[24] S.R. Coleman, V. Glaser, A. Martin, Comm. Math. Phys. 58 (1978) 211-221.

[25] O. Lopes, J. Differential Equations 124 (2) (1996) 378-388.

[26] J. Byeon, L. Jeanjean, M. Mariș, Calc. Var. Partial Differential Equations 36 (4) (2009) 481-492, arXiv:0806.0299 [math.AP].

[27] K. Blum, M. Honda, R. Sato, M. Takimoto, K. Tobioka, J. High Energy Phys. 05 (2017) 109, arXiv:1611.04570 [hep-th]. Erratum:; JHEP 06 (2017) 060.

[28] N. Bar, K. Blum, J. Eby, R. Sato, Phys. Rev. D99 (10) (2019) 103020 , arXiv:1903.03402 [astro-ph.CO]. 